\title{
Declínio da velocidade da marcha e desfechos de saúde em idosos: dados da Rede Fibra
}

\author{
Decreased gait speed and health outcomes in older adults: Rede FIBRA's data \\ Disminución de la velocidad de la marcha y resultados en la salud de los ancianos: datos \\ de la Red Fibra \\ Rita de Cássia Guedes ${ }^{1}$, Rosângela Corrêa Dias ${ }^{2}$, Anita Liberalesso Neri³ ${ }^{3}$ Eduardo Ferriolli", \\ Roberto Alves Lourenço ${ }^{5}$, Lygia Paccini Lustosa ${ }^{6}$
}

\begin{abstract}
RESUMO I A velocidade da marcha (VM) tem sido considerada um marcador de saúde em idosos capaz de predizer desfechos adversos de saúde, mas a compreensão de fatores associados a ela ainda é limitada e controversa. O objetivo deste trabalho é identificar desfechos adversos de saúde relacionados ao declínio da velocidade de marcha em idosos comunitários. Tratase de estudo transversal e multicêntrico, que avaliou o autorrelato de doenças crônicas e de hospitalização no último ano, polifarmácia e velocidade de marcha. Utilizou-se análise de regressão logística para estimar os efeitos de cada variável independente na chance de os idosos apresentarem declínio na velocidade de marcha inferior (VM<0,8m/s) $(\alpha=0,05)$. Participaram da pesquisa 5.501 idosos. A menor velocidade da marcha mostrou-se associada a portadores de doenças cardíacas (OR=2,06; IC: 1,67-2,54), respiratórias (OR=3,25; IC: 2,02$5,29)$, reumáticas $(O R=2,16 ; I C: 1,79-2,52)$ e/ou depressão $(\mathrm{OR}=2,51$; IC: 2,10-3,14), hospitalizados no último ano (OR=1,51; IC: 1,21-1,85) e polifarmácia (OR=2,14; IC: 1,802,54). Assim, os resultados indicaram que idosos com velocidade de marcha menor que $0,8 \mathrm{~m} / \mathrm{s}$ apresentam maior risco de eventos adversos de saúde. Dessa forma, sugere-se que a velocidade de marcha não seja
\end{abstract}

negligenciada na avaliação de idosos comunitários, inclusive na atenção básica.

Descritores I Idoso; Marcha; Doenças Crônicas.

\begin{abstract}
Gait speed (GS) can predict adverse health outcomes. However, an understanding of its associated factors is still limited and with some controversy. The objective of this study was to identify adverse health outcomes related to the decline in gait speed in community-dwelling older adults. This is a cross-sectional study that evaluated records of chronic diseases and hospitalization in the last year, polypharmacy, and gait speed. Logistic regression analysis was used to estimate the effects of each independent variable on the chance of older adults presenting a decline in gait speed (GS<0.8 $\mathrm{m} / \mathrm{s})(\alpha=5 \%)$. In total, 5,501 older adults participated. Brazilian older adults with heart diseases ( $\mathrm{OR}=2.06$; 1.67$2.54 \mathrm{Cl})$, respiratory diseases $(\mathrm{OR}=3.25 ; 2.02-5.29 \mathrm{Cl})$, rheumatic $(\mathrm{OR}=2.16 ; 1.79-2.52 \mathrm{Cl})$ and/ or depression diseases $(\mathrm{OR}=2.51 ; 2.10-3.14 \mathrm{Cl})$; hospitalized in the last year $(\mathrm{OR}=1.51 ; 1.21-1.85 \mathrm{Cl})$ and under polypharmacy $(\mathrm{OR}=2.14 ; 1.80-2.54 \mathrm{Cl}$ ) were associated with lower gait speed. Thus, the results showed that those with gait speed lower than $0.8 \mathrm{~m} / \mathrm{s}$ are at higher risk of some adverse
\end{abstract}

Estudo multicêntrico realizado em 17 cidades brasileiras por pesquisadores da Universidade Federal de Minas Gerais, Universidade de São Paulo, Universidade de Campinas e Universidade do Estado do Rio de Janeiro.

'Centro Universitário de Belo Horizonte (UniBH) - Belo Horizonte (MG), Brasil. E-mail: rita.guedes@unibh.br.

Orcid: 0000-0001-8490-1882

2Universidade Federal de Minas Gerais (UFMG) - Belo Horizonte (MG), Brasil. E-mail: rcorreadias8@gmail.com.

Orcid: 0000-0002-1027-7746

3 Universidade Estadual de Campinas (Unicamp) - Campinas (SP), Brasil. E-mail: anitalbn@uol.com.br. Orcid: 0000-0002-6833-7668

${ }^{4}$ Universidade de São Paulo (USP) - Ribeirão Preto (SP), Brasil. E-mail: eferriol@fmrp.usp.br. Orcid: 0000-0002-5028-2451

5Universidade do Estado do Rio de Janeiro (Uerj) - Rio de Janeiro (RJ), Brasil. E-mail: roberto.lourenco@globo.com.

Orcid: 0000-0003-0838-1285

6Universidade Federal de Minas Gerais (UFMG) - Belo Horizonte (MG), Brasil. E-mail: Iygiapl@ufmg.br. Orcid: 0000-0002-0919-1320 
health events. Therefore, it is suggested that gait speed should not be neglected in the evaluation in community-dwelling older adults, including basic health care.

Keywords | Aged; Gait; Chronic Diseases.

RESUMEN | La velocidad de la marcha (VM) se ha considerado un marcador de salud en los ancianos capaz de predecir resultados adversos en la salud, pero la comprensión de sus factores asociados todavía es limitada y controvertida. El presente estudio tiene como objetivo identificar los resultados adversos para la salud relacionados con la disminución de la velocidad de la marcha en los ancianos comunitarios. Se trata de un estudio transversal y multicéntrico, el que evaluó el autoinforme de enfermedades crónicas y de la hospitalización en el último año, la polifarmacia y la velocidad de la marcha. Se utilizó el análisis de regresión logística para estimar los efectos de cada variable independiente sobre la posibilidad de que los ancianos presenten una disminución más baja en la velocidad de la marcha ( $\mathrm{VM}<0,8 \mathrm{~m} / \mathrm{s})(\alpha=0,05)$. Participaron en el estudio 5.501 ancianos. La velocidad de marcha más baja estuvo asociada a portadores de enfermedades cardíacas (OR=2,06; IC: 1,67-2,54), respiratorias (OR=3,25; IC: 2,02-5,29), reumáticas (OR=2,16; IC: 1,79-2,52) y/o depresión (OR=2,51; IC: 2,10-3,14), hospitalizados en el último año (OR=1,51; IC: 1,21-1,85) y polifarmacia (OR=2,14; IC: 1,80-2,54). De esta manera, los resultados indicaron que los ancianos con velocidades de marcha por debajo de $0,8 \mathrm{~m} / \mathrm{s}$ presentan un mayor riesgo de eventos adversos para la salud. Por lo tanto, se sugiere que la velocidad de la marcha no debe ser descuidada en la evaluación de ancianos comunitarios, incluso en la atención primaria.

Palabras clave Anciano; Marcha; Enfermedades Crónicas.

\section{INTRODUÇÃO}

A função física diminui com a idade e prediz maiores taxas de incapacidade, institucionalização e morte. Assim, a manutenção da capacidade funcional dos idosos é extremamente importante no prolongamento da vida independente ${ }^{1,2}$. O acúmulo de doenças influencia de forma negativa a capacidade funcional e a qualidade de vida ${ }^{3,4}$. No contexto da capacidade funcional, a marcha consiste em uma atividade dinâmica fundamental para a realização das atividades de vida diária e necessária para a independência dos indivíduos ${ }^{5}$. No entanto, com o envelhecer, a marcha tende a se tornar lenta, sugerindo que cada indivíduo seleciona a velocidade mais compatível às suas capacidades funcionais. Assim, a lentidão da marcha surge como consequência do processo fisiológico do envelhecimento, muitas vezes associada a condições clínicas já instaladas ou àquelas que ainda não se manifestaram, mantendo-se silenciosas no quadro clínico do idoso ${ }^{6}$.

Nesse contexto, a velocidade de marcha habitual tem sido considerada um preditor de declínio funcional, hospitalização, alta hospitalar, necessidade de cuidador e mortalidade ${ }^{7}$. Da mesma forma, ela tem se mostrado associada com maior incidência de fraturas, institucionalização e mudanças na qualidade de vida, fatores que aumentam os custos com a saúde ${ }^{8,9}$. Neste contexto, observou-se que a redução de $0,1 \mathrm{~m} / \mathrm{s}$ na velocidade de marcha aumenta em $12 \%$ o risco de morte ${ }^{7,10}$ e que idosos com declínio na velocidade da marcha demonstraram 2,5 vezes maior chance de apresentarem desfechos adversos de saúde ${ }^{11}$. Assim, por ser um importante indicador de saúde e função, e ser facilmente avaliada e interpretada, a velocidade de marcha tem sido considerada como "sexto sinal vital" e um marcador funcional de saúde ${ }^{6}$.

A Academia Internacional de Nutrição e Envelhecimento identificou diversos pontos de corte para a velocidade de marcha, variando entre $0,15 \mathrm{~m} / \mathrm{s}$ e $1,3 \mathrm{~m} / \mathrm{s}$, relacionados a desfechos adversos de saúde, entre outros, como quedas, doenças cardíacas e pulmonares, fragilidade, sarcopenia, hospitalização, institucionalização e morte ${ }^{8}$. Em particular, Studenski et al. ${ }^{7}$ utilizaram o ponto de corte de $0,8 \mathrm{~m} / \mathrm{s}$ e demonstraram que idosos com marcha mais rápida apresentavam maior expectativa de vida. Além disso, presença de doenças crônicas, polifarmácia, baixas condições sociais e educacionais também têm sido relacionadas com declínio da velocidade de marcha, mobilidade e piores prognósticos de saúde ${ }^{3,5,8,11}$. No entanto, ainda é limitada a compreensão de seus fatores associados e suas implicações em diversas populações. Estudo recente demonstrou a influência de fatores econômicos, sociais e expectativa de vida ao nascer com as alterações da velocidade de marcha, considerando os domínios do Índice de Desenvolvimento Humano (IDH) ${ }^{12}$.

Assim, o objetivo do presente estudo foi determinar o risco de desfechos adversos de saúde (doenças cardíacas, respiratórias e reumáticas; hipertensão arterial sistêmica; diabetes mellitus; câncer; osteoporose; depressão; 
hospitalização e polifarmácia) estarem associados ao declínio da velocidade de marcha em idosos brasileiros que vivem na comunidade.

\section{METODOLOGIA}

Trata-se de um estudo transversal, multicêntrico e que é parte do projeto Rede de Estudos de Fragilidade em Idosos Brasileiros (Fibra). Esta rede multidisciplinar avaliou idosos residentes em diferentes cidades das cinco regiões brasileiras, selecionados por meio de um processo de aleatorização em regiões censitárias. Os procedimentos de seleção de amostra já foram publicados em estudos prévios, assim como os critérios de inclusão/exclusão ${ }^{13,14}$. Dessa forma, foram convidados a participar do estudo indivíduos com mais de 65 anos, residentes na comunidade e que concordassem em participar da entrevista e dos testes físicos por meio de Termo de Consentimento Livre e Esclarecido ${ }^{13}$. Os dados foram coletados no domicílio dos participantes, em entrevistas previamente agendadas e por pesquisadores treinados. Este estudo seguiu as diretrizes do strengthening the reporting of observational studies in epidemiology (Strobe).

Os critérios de exclusão foram: apresentar sequelas graves de acidente vascular encefálico e/ou doenças neurológicas que impedissem a realização dos testes; usar cadeira de rodas ou estar acamado; e apresentar déficit cognitivo indicado pela pontuação inferior a 17 no miniexame do estado mental ${ }^{15}$. Para atender aos objetivos deste estudo foram realizadas análises do banco nacional da Rede Fibra, contendo informações coletadas em todas as cidades participantes.

A variável dependente foi a velocidade de marcha habitual (m/s), avaliada na distância de 4,6m, acrescidos de $2 \mathrm{~m}$ de aceleração e $2 \mathrm{~m}$ para desaceleração. Os participantes usaram seu calçado habitual. Os idosos foram orientados a andar na sua velocidade normal, a partir do comando de voz de "pronto? Vai..." O tempo de deslocamento foi, então, cronometrado. Foram feitas três medidas, e utilizada a média para as análises. A velocidade de marcha foi usada como variável categórica, utilizando-se o ponto de corte de $0,8 \mathrm{~m} / \mathrm{s}$, indicado como o valor mais sensível para identificar a influência dos

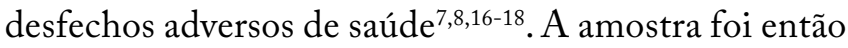
categorizada em um grupo composto por indivíduos com velocidade de marcha inferior a $0,8 \mathrm{~m} / \mathrm{s}$ e outro grupo formado por participantes com velocidade de marcha igual ou superior a $0,8 \mathrm{~m} / \mathrm{s}$.
As variáveis independentes foram avaliadas por inquérito multidimensional padronizado pela Rede Fibra, por meio de entrevista e informações autorrelatadas. Essa metodologia foi utilizada nos trabalhos da Rede Fibra $^{13,14}$. Para o presente trabalho foram selecionadas as seguintes variáveis: autorrelato de presença de doenças crônicas diagnosticadas por um médico (doenças cardíacas, respiratórias e reumáticas; hipertensão arterial sistêmica; diabetes mellitus; câncer, osteoporose e depressão) ("alguma vez o seu médico lhe informou que o(a) senhor(a) tinha...?”); autorrelato de hospitalização nos últimos 12 meses ("o(a) senhor(a) esteve internado(a) nos últimos 12 meses?”) e polifarmácia (definida como o uso de quatro ou mais medicamentos regulares durante os últimos três meses, por meio de consulta às embalagens dos medicamentos e/ou prescrição).

\section{Análise estatística}

A pesquisa baseou-se em uma amostra probabilística de idosos comunitários, estratificada segundo a densidade populacional de idosos, com base nos dados do censo 2000 realizado pelo Instituto Brasileiro de Geografia e Estatística. Para o cálculo da amostra de cada cidade foi estimado o tamanho amostral necessário para uma proporção populacional de $50 \%$ de uma determinada característica em estudo (valor em que o tamanho amostral obtido foi o máximo possível ( $p=0,50 ; q=0,50)$, conforme a população idosa de cada uma delas). Foi fixado o nível de significância de 5\% $(\mathrm{alfa}=0,05 ; Z=1,96)$. O erro amostral variou entre $3 \%$ e $5 \%$. Com base nesses cálculos, o tamanho amostral para as cidades com mais de um milhão de habitantes foi fixado em 601 idosos, para um erro de 4\%. Para as cidades com menos de um milhão de habitantes, o valor da amostra foi fixado em 385 indivíduos, correspondente a um erro de $5 \%$.

A descrição da amostra foi dada por medidas de média e desvio-padrão para as variáveis contínuas, e de porcentagem para as variáveis categóricas. A análise de distribuição dos dados foi feita por meio do teste Kolmogorov-Smirnov. Diferenças entre grupos foram avaliadas pelos testes Kruskall-Wallis e qui-quadrado. Variáveis com valor-p maior que 0,10 nas análises bivariadas não foram incluídas nas análises multivariadas. Foi traçado modelo de regressão logística binária, stepwise forward, tendo como variável dependente a velocidade de marcha (categorizada em menor que $0,8 \mathrm{~m} / \mathrm{s}$ e igual ou maior que $0,8 \mathrm{~m} / \mathrm{s}$ ) e como variáveis 
independentes (autorrelato de presença de doenças cardíacas, respiratórias e reumáticas; hipertensão arterial sistêmica; diabetes mellitus; câncer, osteoporose e depressão, diagnosticados por médico; hospitalização e polifarmácia). Dessa forma, esta análise teve como objetivo explorar a associação de risco das variáveis independentes com a variável dependente (velocidade de marcha menor que $0,8 \mathrm{~m} / \mathrm{s}$ quando comparado com idosos com velocidade de marcha igual ou maior que $0,8 \mathrm{~m} / \mathrm{s}$ ). A chance de cada variável independente se associar com a velocidade de marcha foi analisada pela odds ratio (OR) e pelo intervalo de confiança (IC) $95 \%$. Variáveis sociodemográficas não foram incluídas no modelo por não serem o objetivo deste estudo. Todas as análises foram realizadas no programa estatístico SPSS 17.0 e foi considerado nível de significância de $5 \%$.

\section{RESULTADOS}

Participaram deste estudo 5.501 idosos de todo o Brasil (Rede Fibra). A maioria eram mulheres $(66,20 \%)$ e a média de idade foi de 73,01 $\pm 6,17$ anos. A média global da velocidade de marcha foi $1,00 \pm 0,69 \mathrm{~m} / \mathrm{s}$, sendo que $34,26 \%$ dos idosos apresentaram $\mathrm{VM}<0,8 \mathrm{~m} / \mathrm{s}$.
Assim, os idosos classificados neste grupo, com $\mathrm{VM}<0,8 \mathrm{~m} / \mathrm{s}$, apresentaram maior relato de doenças crônicas. As análises univariadas mostraram diferenças estatisticamente significantes entre os idosos com $\mathrm{VM}<0,8 \mathrm{~m} / \mathrm{s}$ e com $\mathrm{VM} \geq 0,8 \mathrm{~m} / \mathrm{s}$, em relação a todas as variáveis independentes $(\mathrm{p}<0,05)$, exceto aqueles com relato de câncer. Estes resultados e as demais características da amostra encontram-se na Tabela 1.

Para as análises multivariadas, as variáveis com valor-p maior que 0,10 não foram incluídas no modelo. Assim, o modelo que melhor explicou a velocidade de marcha identificou que idosos que autorrelataram terem recebido diagnóstico médico de doenças cardíacas, doenças respiratórias, doenças reumáticas e/ou depressão; que autorrelataram terem sido hospitalizados nos últimos 12 meses e que faziam uso regular de quatro ou mais medicamentos nos últimos três meses demonstraram maior chance de ter $\mathrm{VM}<0,8 \mathrm{~m} / \mathrm{s}$. Neste caso, na presença destas condições os resultados demonstraram pelo menos 1,51 chance maior dos idosos apresentarem o declínio da velocidade de marcha (Tabela 2). Autorrelato de hipertensão arterial sistêmica, diabetes mellitus, câncer e osteoporose, mesmo que inicialmente se mostrassem diferentes, não permaneceram no modelo final.

Tabela 1. Média, desvio-padrão e frequência de distribuição das variáveis independentes, comparando idosos com VM<0,8m/s e aqueles com $\vee M \geq 0,8 m / s$ e valor-p

\begin{tabular}{|c|c|c|c|c|}
\hline Características & Amostra total $(\mathrm{N}=5501)$ & Idosos VM<0,8m/s (N=1885) & Idosos VM $\geq 0,8 \mathrm{~m} / \mathrm{s} \quad(\mathrm{N}=3616)$ & valor-p \\
\hline Idade (anos), média \pm DP & $73,01 \pm 6,17$ & $75,11 \pm 6,77$ & $72,18 \pm 5,65$ & $<0,01^{*}$ \\
\hline Sexo & & & & $<0,01^{*}$ \\
\hline Masculino, n (\%) & $1862(33,81)$ & $476(25,30)$ & $1386(38,30)$ & \\
\hline Feminino, $\mathrm{n}(\%)$ & $3639(66,20)$ & $1409(74,70)$ & $2230(61,70)$ & \\
\hline Doenças cardíacas, n (\%) & $1005(18,30)$ & $590(31,30)$ & $415(11,50)$ & $<0,01^{*}$ \\
\hline Doenças respiratórias, n (\%) & $435(7,90)$ & $385(20,40)$ & $50(1,40)$ & $<0,01^{*}$ \\
\hline Doenças reumáticas, n (\%) & $1738(31,60)$ & $852(45,20)$ & $886(24,50)$ & $<0,01^{*}$ \\
\hline HAS, n (\%) & $3173(57,70)$ & $1127(59,80)$ & $2046(56,60)$ & $0,02^{*}$ \\
\hline Diabetes mellitus, n (\%) & $1079(19,60)$ & $665(18,40)$ & $414(22,00)$ & $<0,01^{*}$ \\
\hline Câncer, n (\%) & $260(4,70)$ & $79(4,20)$ & $181(5,00)$ & 0,09 \\
\hline Osteoporose, n (\%) & $1183(21,50)$ & $457(24,30)$ & $726(20,10)$ & $<0,01^{*}$ \\
\hline Depressão, n (\%) & $908(16,50)$ & $352(18,70)$ & $556(15,40)$ & $0,02^{*}$ \\
\hline $\begin{array}{l}\text { Internação hospitalar no } \\
\text { último ano, n (\%) }\end{array}$ & $893(16,20)$ & $437(23,20)$ & $456(12,60)$ & $<0,01^{*}$ \\
\hline Polifarmácia, n (\%) & $1719(31,24)$ & $852(45,20)$ & $867(24,00)$ & $<0,01^{*}$ \\
\hline VM (m/s), média $\pm \mathrm{DP}$ & $1,0 \pm 0,69$ & $0,59 \pm 0,15$ & $1,06 \pm 0,19$ & $<0,01^{*}$ \\
\hline
\end{tabular}


Tabela 2. Razão de chance, intervalo de confiança e significância estatística dos fatores associados à VM inferior a $0,8 \mathrm{~m} / \mathrm{s}$, na amostra de idosos da Rede Fibra

\begin{tabular}{lccc}
\multicolumn{1}{c}{ VARIÁVEIS } & OR & IC 95\% & Valor-p \\
Doenças cardíacas & 2,06 & $(1,67-2,54)$ & $<0,01^{*}$ \\
Doenças respiratórias & 3,25 & $(2,02-5,29)$ & $0,01^{*}$ \\
Doenças reumáticas & 2,16 & $(1,79-2,52)$ & $<0,01^{*}$ \\
Depressão & 2,51 & $(2,10-3,14)$ & $<0,01^{*}$ \\
Hospitalização & 1,51 & $(1,21-1,85)$ & $<0,01^{*}$ \\
Polifarmácia & 2,14 & $(1,80-2,54)$ & $<0,01^{*}$
\end{tabular}

VM: velocidade de marcha habitual; IC95\%: intervalo de confiança de $95 \%$ de confiança; OR: odds ratio; *diferença significativa.

\section{DISCUSSÃO}

Este estudo teve como objetivo analisar a relação de alguns desfechos adversos de saúde (doenças cardíacas, respiratórias e reumáticas; hipertensão arterial sistêmica; diabetes mellitus; câncer, osteoporose, depressão, hospitalização e medicamentos) com o declínio da velocidade de marcha em idosos. Os resultados demonstraram que aqueles com $\mathrm{VM}<0,8 \mathrm{~m} / \mathrm{s}$, apresentaram maior histórico de hospitalização, de algumas condições clínicas e de maior uso de fármacos. Esses resultados confirmam a literatura nacional e internacional, e apontam para a diminuição de reservas de energia e perda da homeostase no processo de envelhecimento ${ }^{1-6}$.

Os idosos com diagnóstico de doenças cardíacas como angina, infarto do miocárdio ou insuficiência cardíaca, apresentaram 2,06 vezes maior chance de ter $\mathrm{VM}<0,8 \mathrm{~m} / \mathrm{s}$. Uma das justificativas para esta forte associação poderia ser o fato de as doenças crônicas, de forma geral, levarem à redução de massa muscular como consequência de processos fisiopatológicos que aumentam o catabolismo celular ${ }^{19}$. Da mesma forma, a literatura aponta que sintomas respiratórios e mobilidade reduzida, assim como as doenças cardíacas, estão presentes na velhice e associados com importantes desfechos de saúde ${ }^{20}$. Nossos resultados demonstraram também que aqueles com diagnóstico de bronquite e/ ou enfisema apresentaram 3,25 vezes maior chance de ter $\mathrm{VM}<0,8 \mathrm{~m} / \mathrm{s}$. Neste contexto, estudos prévios confirmaram o presente resultado, identificando associação da redução da velocidade de marcha com o aumento da gravidade das doenças pulmonares e forte correlação negativa entre a velocidade de marcha e severidade da dispnéia ${ }^{21}$. Esses resultados podem ser justificados pela restrição e diminuição da capacidade aeróbia na presença destas condições.

Inserido ainda no contexto das doenças crônicogenerativas, sabe-se que as doenças reumáticas também estão associadas ao declínio funcional e à lentidão da marcha em idosos ${ }^{22}$. Neste caso, a redução da velocidade de marcha pode ser interpretada como consequência da fraqueza muscular nos membros inferiores e das alterações proprioceptivas que comprometem o equilíbrio postural do indivíduo, na presença destas doenças. Além disso, existem evidências que, na presença de doenças articulares e reumáticas, o indivíduo tende a diminuir a cadência e o tamanho do passo e passada, como estratégia para reduzir as forças compressivas nas articulações dos membros inferiores e, consequentemente, aliviar a dor ${ }^{22,23}$. Esses resultados estão de acordo com pesquisas que analisaram os parâmetros específicos da marcha ${ }^{12,22,23}$.

Idosos com maior número de sintomas depressivos demonstraram 2,51 vezes maior chance de caminhar em $\mathrm{VM}<0,8 \mathrm{~m} / \mathrm{s}$. Este resultado confirmou a literatura que aponta que depressão e lentidão da marcha, assim como outras condições crônico-degenerativas discutidas anteriormente, acontecem com grande frequência e simultaneamente na velhice. Novamente, essas condições estão associadas a incapacidades, declínio cognitivo, fatores ambientais e psicossociais que podem influenciar no processo de envelhecimento saudável ${ }^{24-26}$. Os resultados apresentados aqui não permitem inferir sobre causalidade, mas apresentam relevância ao demonstrar associação entre estas condições, confirmando que a VM pode ser considerada um marcador de saúde funcional.

Idosos com maior número de condições crônicodegenerativas tendem a utilizar um maior número de medicamentos, que também podem ter impacto na saúde global e funcional ${ }^{27}$. Este pressuposto também foi confirmado neste estudo, que apontou que aqueles que faziam uso regular de quatro ou mais medicamentos apresentaram 2,14 maior chance ter a $\mathrm{VM}<0,8 \mathrm{~m} / \mathrm{s}$. Peron et $\mathrm{al} .{ }^{28}$ demonstraram que o uso de medicamentos como os benzodiazepínicos, anticolinérgicos, antidepressivos e anti-hipertensivos estiveram associados com o pior estado funcional. Neste estudo, não foi feita uma análise específica do tipo de fármaco utilizado pelos idosos, apenas em relação ao número de medicações em uso, o que pode ser considerado uma limitação e deve ser explorado em pesquisas futuras. 
Idosos que relataram ter sido hospitalizado nos últimos 12 meses apresentaram 1,51 vezes maior chance de andar em $\mathrm{VM}<0,8 \mathrm{~m} / \mathrm{s}$. É sabido que após a alta hospitalar,os pacientes frequentemente experimentam consequências negativas a curto, médio e longo prazo, como declínio funcional, incapacidades, nova internação, institucionalização e morte $^{26}$. Estudos recentes demonstraram que idosos reduzem a capacidade funcional durante o período de hospitalização e não conseguem recuperá-la após a alta hospitalar ${ }^{29}$,chegando a $19 \%$ com limitações nas atividades básicas de vida diária e 40\% nas atividades instrumentais de vida diária, após a hospitalização ${ }^{29,30}$. Neste caso, não foram analisadas o número de internações e o seu motivo, o que pode ser uma limitação na interpretação deste dado. Da mesma forma, é importante relatar, em estudos futuros, o tempo de diagnóstico das doenças, pois existem indícios de que uma maior restrição da mobilidade e capacidade funcional pode estar associada com o agravamento e cronificação das doenças.

Um dado importante a ser discutido refere-se à diferença significativa observada entre os grupos em relação à idade. Este estudo não teve como objetivo explorar variáveis sociodemográficas, apesar de os autores reconhecerem as evidências desta relação ${ }^{12}$. Inclusive a idade apresenta multicolinearidade, principalmente em relação às doenças cardíaca, respiratórias, reumáticas e hospitalização, não justificando sua análise no modelo. Neste contexto, sabe-se que a marcha e seus parâmetros espaçotemporais tendem a se modificar com o passar dos anos e com algumas doenças ${ }^{5,6,31}$. Alguns autores demonstraram uma perda fisiológica, incluindo a velocidade da marcha, inerente ao processo de envelhecimento, ou seja, com o passar dos anos de vida ${ }^{12,31}$. Desta forma, diante da multicolinearidade da idade com as variáveis independentes analisadas, sugerese realizar estudos futuros, com análises longitudinais e de mediação, para verificar a magnitude da influência da idade.

Finalmente, os dados apresentados aqui demonstraram relevância por ser um estudo multicêntrico, assegurando participação de idosos das cinco regiões do país e possibilidade de conhecer esta característica da população estudada. Como a literatura aponta, a velocidade de marcha pode sofrer influência de fatores contextuais, socioeconômicos, clínicos e de acesso a serviços de saúde ${ }^{12}$. No caso do Brasil, que apresenta dimensões continentais e grande diversidade regional, este estudo é o primeiro passo para compreender melhor o comportamento da marcha dos idosos moradores da comunidade, o que pode subsidiar políticas de saúde para esta população e outras com características semelhantes. Dessa forma, do ponto de vista de aplicabilidade clínica, os resultados encontrados neste estudo sugerem que a velocidade de marcha pode ser considerada um marcador de saúde para idosos comunitários. Sugere-se, assim, o uso desta medida na prática clínica e maior atenção àqueles que apresentarem maior lentidão na marcha.

\section{CONCLUSÃO}

Os resultados do presente estudo indicaram que os idosos avaliados, com velocidade de marcha menor que $0,8 \mathrm{~m} / \mathrm{s}$, estão em maior risco de eventos adversos de saúde (doenças cardíacas, respiratórias e reumáticas; hipertensão arterial sistêmica; diabetes mellitus; câncer; osteoporose; depressão; hospitalização e número de medicamentos). Dessa forma, sugere-se que a velocidade de marcha não deve ser negligenciada na avaliação do idoso, mesmo na atenção básica.

\section{REFERÊNCIAS}

1. Cooper R, Kuh D, Cooper C, Gale CR, Lawlor DA, Matthews F, et al. Objective measures of physical capability and subsequent health: a systematic review. Age Ageing. 2011;40(1):14-23. doi: 10.1093/ageing/afq117

2. Andrade JM, Duarte YAO, Alves LC, Andrade FCD, Souza Junior PRB, Lima-Costa MF, et al. Frailty profile in Brazilian older adults: ELZI-Brazil. Rev Saúde Publica. 2018;52(2):1-17. doi: 10.11606/ S1518-8787.20180520000616

3. Nunes BP, Batista SRR, Andrade FB, Souza Junior PRB, LimaCosta MF, Facchini LA. Multimorbidity: the Brazilian longitudinal study of aging (ELSI-Brazil). Rev Saúde Publica. 2018;52(2):10. doi: 10.11606/S1518-8787.20180520000637

4. Montero-Odasso M, Verghese J, Beauchet O, Hausdorff JM. Gait and cognition: a complementary approach to understanding brain function and the risk of falling. J Am Geriatr Soc. 2012;60(11):2127-36. doi: 10.1111/j.1532-5415.2012.04209.x

5. Fiser WM, Hays NP, Rogers SC, Kajkenova o, Williams AE, Evans CM, et al. Energetics of walking in elderly people: factors related to gait speed. J Gerontol A Biol Sci Med Sci. 2010;65(12):1332-7. doi: 10.1093/gerona/glq137

6. Studenski S. Bradypedia: is gait speed ready for clinical use? J Nutr Health Aging. 2009;13(10):878-80.

7. Studenski S, Perera S, Patel K, Rosano c, Faulkner K, Inzitari m, et al. Gait speed and survival in older adults. JAMA. 2011;305(1):508. doi: 10.1001/jama.2010.1923

8. van Kan Ga, Rolland Y, Andrieu S, Bauer J, Beauchet O, Cesari $M$, et al. Gait speed at usual pace as a predictor of adverse outcomes in community-dwelling older people an International Academy on Nutrition and Aging (IANA) Task Force. J Nutr Health Aging. 2009;13(10):881-9. 
9. Fritz S, Lusardi M. White paper: "walking speed: the sixth vital sign”. J Geriatr Phys Ther. 2009;32(2):46-9.

10. Pinter D, Ritchie SJ, Gattringer T, Bastin ME, Corley J, Pattie A, et al. Predictors of gait speed and its change over three years in community-dwelling older people. Aging. 2018;10(1):144-53. doi: 10.18632/aging.101365

11. Montero-Odasso M, Schapira M, Soriano ER, Varela M, Keplan R, Camera $L A$, et al. Gait velocity as a single predictor of adverse events in healthy seniors aged 75 years and older. J A Gerontol Biol Sci Med Sci. 2005;60(10):1304-9.

12. Freire Jr RC, Pieruccini-Faria F, Montero-Odasso M. Are human development index dimensions associated with gait performance in older adults? A systematic review. Exper Gerontol. 2018;102:59-68. doi: 10.1016/j.exger.2017.12.001

13. Silva SLA, Neri AL, Ferrioli E, Lourenço RA, Dias RC. Phenotype of frailty: the influence of each item in determining frailty in community-dwelling elderly - The Fibra Study. Cienc Saude Coletiva. 2016;21(11):3483-92. doi: 10.1590/1413-812320152111.23292015

14. Moreira BS, Sampaio RF, Diz JB, Bastone AC, Ferrioli E, Neri AL et al. Factors associated with fear of falling in community-dwelling older adults with and without diabetes mellitus: findings from the Frailty in Brazilian Older People Study (FIBRA-BR). Exp Gerontol. 2017;89:103-11. doi: 10.1016/j.exger.2017.01.004

15. Brucki SMD, Nitrini R, Caramelli P, Bertolucci PHF, Okamoto IH. Suggestions for utilization of the mini-mental state examination in Brazil. Arq Neuro-Psiquiatr. 2003;61(3B):777-81. doi: 10.1590/ S0004-282X2003000500014

16. Cruz-Jentoft AJ, Bahat G, Bauer J, Boirie Y, Bruyère O, Landi F, et al. Sarcopenia: revised European consensus on definition and diagnosis. Age Ageing. 2019;48(1):16-31. doi: 10.1093/ageing/ afy169

17. Cesari M. Role of gait speed in the assessment of older patients. JAMA. 2011;305(1):93-4. doi: 10.1001/jama.2010.1970

18. Pamoukdjian F, Paillard E, Zelek L, Laurent M, Lévy V, Landre $T$, et al. Measurement of gait speed in older adults to identify complications associated with frailty: a systematic review. J Geriatr Oncol. 2015;6(6):484-96. doi: 10.1016/j.jgo.2015.08.006

19. Fearon K, Evans WJ, Anker SD. Myopenia-a new universal term for muscle wasting. J Cachexia Sarcopenia Muscle. 2011;2(1):1-3.

20. Gill TM, Gahbauer EA, Murphy TE, Han L, Allore HG. Risk factors and precipitants of long-term disability in community mobility: a cohort study of older persons. Ann Intern Med. 2012;156(2):13140. doi: 10.7326/0003-4819-156-2-201201170-00009

21. Ilgin D, Ozalevli S, Kilinc O, Sevinc C, Cimrin AH, Ucan ES. Gait speed as a functional capacity indicator in patients with chronic obstructive pulmonary disease. Ann Thoracic Med. 2011;6(3):141-6. doi: 10.4103/1817-1737.82448

22. White DK, Niu J, Zhang Y. Is symptomatic knee osteoarthritis a risk factor for a trajectory of fast decline in gait speed? Results from a longitudinal cohort study. Arthritis Care Res. 2013;65(2):187-94. doi: 10.1002/acr.21816

23. White DK, Felson DT, Niu J, Nevitt MC, Lewis CE, Torner JC. Reasons for functional decline despite reductions in knee pain: the Multicenter Osteoarthritis Study. Phys Ther. 2011;91(12):184956. doi: 10.2522/ptj.20100385

24. Demakakos P, Cooper R, Hamer M, Oliveira C, Hardy R, Breeze $\mathrm{E}$. The bidirectional association between depressive symptoms and gait speed: evidence from the English Longitudinal Study of Ageing (ELSA). PloS One. 2013;8(7):e68632. doi: 10.1371/ journal.pone.0068632

25. Parodi JF, Nieto-Gutierrez W, Tellez WA, Ventocilla-Gonzales I, Runzer-Colmenares FM, Taype-Rondan A. Gait speed and the appearance of neurocognitive disorders in older adults: results of a Peruvian cohort. Rev Esp Geriatr Gerontol. 2018;53(2):73-6. doi: 10.1016/j.regg.2017.08.001

26. Sanders JB, Bremmer MA, Deeg DJ, Beekman AT. Do depressive symptoms and gait speed impairment predict each other's incidence? A 16-year prospective study in the community. J Am Geriatr Soc. 2012;60(9):1673-80. doi: 10.1111/j.1532-5415.2012.04114.x

27. Morandi A, Bellelli G, Vasilevskis EE, Turco R, Gentile S, Guerini F. Predictors of rehospitalization among elderly patients admitted to a rehabilitation hospital: the role of polypharmacy, functional status, and length of stay. J Am Med Dir Ass. 2013;14(10):761-7. doi: 10.1016/j.jamda.2013.03.013

28. Peron EP, Gray SL, Hanlon JT. Medication use and functional status decline in older adults: a narrative review. Am J Geriatr Pharmacother. 2011;9(6):378-91. doi: 10.1016/j. amjopharm.2011.10.002

29. Volpato S, Cavalieri M, Sioulis F, Guerra G, Maraldi C, Zuliani G, et al. Predictive value of the short physical performance battery following hospitalization in older patients. J Gerontol A Biol Sci Med Sci. 2011;66(1):89-96. doi: 10.1093/gerona/glq167

30. Barthuly AM, Bohannon RW, Gorack W. Gait speed is a responsive measure of physical performance for patients undergoing short-term rehabilitation. Gait Posture. 2012;36(1):61-4. doi: 10.1016/j.gaitpost.2012.01.002

31. Ghaffar A, Dehghani-Sanij AA, Xie SQ. A review of gait disorders in the elderly and neurological patients for robotassisted training. Disabil Rehabil Assist Technol. 2019:1-15. doi: 10.1080/17483107.2019.1568594 